\title{
6 Conclusions and implications for management and policy
}

This concluding chapter spells out the overall importance of the analyses to the three theory traditions that motivate the book-namely innovation studies, transition studies, and S\&TS - and in doing so elaborates on citizen activities in long-term sociotechnical change. It further reflects on the application and development of the BOAP approach and draws implications for innovation, energy, and climate policies.

The book has linked theoretically and empirically informed sub-studies to a gradual grounded theorization, which can then be contrasted and compared to both sub-study findings and study framings, and onward to more generallevel models of sociotechnical change. We argue that this mid-range BOAP investigation strategy offers a better empirical and theoretical grounding to our view of users in sociotechnical change than has hitherto been available. Let us first examine what it offers for the intersection of innovation studies and S\&TS regarding S-RET innovation and community involvement and then move on to the intersection between S\&TS and transitions regarding user activities in sociotechnical change.

\subsection{The intersection of innovation studies and S\&TS: from local innovation and energy communities to hybrid Internet communities that aid wide diffusion}

Communities in their various forms are known to be key enablers and catalysts of citizen pursuits directed toward alternative technology. Innovation studies features an abundance of literature on innovation communities by users, as well as those hosted by companies (e.g., Jeppesen and Molin, 2003; von Hippel, 2005, 2016; Marchi et al. 2010), while S\&TS has had a more direct focus on energy technologies through research on technology-oriented grassroots innovation movements (e.g., Hess, 2016; Ornetzeder and Rohracher, 2013; Smith et al., 2016a) and community energy (Walker and Devine-Wright, 2008; Hargrieves et al., 2013; Smith et al., 2016b; Creamer et al., 2019). Most of this work examines communities as catalysts for innovation and anchors the discussion of communities to shared locality, or its digital surrogate, in which participants share a similar topical orientation, if not a joint mission, as in open-source projects.

DOI: $10.4324 / 9781003133919$ 
These innovation communities and citizen initiatives such as community energy have been found important in the initial invention and proliferation of alternative technologies. Be it solar collectors in Austria (Ornetzeder and Rohracher, 2006), wind turbines in Denmark (Karnøe and Garud, 2012; Nielsen, 2016), or mountain bikes, rodeo kayaks, and kite surfing equipment in their early venues (Lüthje, 2004; Lüthje et al., 2005; Hienert, 2006; von Hippel, 2005), it has been the early user innovators and their close peers that have generated a new type of alternative technology, initial practices of use, and new sets of meanings. These have then proliferated to other like-minded communities and often resulted in increasing adopter and community participant numbers, as well as further inventions (Ornetzeder and Rohracher, 2013; Hienert, 2006; Hienert et al., 2014). Furthermore, some of the alternative innovations have become mainstream through the commercialization of activities - a pattern found from innovations related to scientific instruments to the related hardware and software in enthusiast communities (von Hippel, 2005; Flichy, 2007; Williams et al., 2005) and in S-RET development as well (Ornetzeder and Rohracher, 2013). At the same time alternative routes to sociotechnical change have been proposed through the peer diffusion (von Hippel, 2016; Hyysalo and Usenyuk, 2015), mainstreaming, replication, and critical niche development of alternative technologies (Smith et al., 2016b; Kohtala, 2017).

Our analyses of S-RET user-innovation and user-run internet communities at once lend support to the above basic schemata regarding the early years of sociotechnical change. However, the analyses also show that the above view overlooks continued innovation by users in the later stages of sociotechnical change, the social value this creates, and the key differences there are between diverse and wide-reaching digital communities and local (or otherwise focused) innovation communities.

\subsubsection{Continued innovation by users in the later stages of sociotechnical change and the social value this creates}

That over 200 S-RET innovations were identified well after the early stages of the global and even local proliferation of the studied S-RETs poses the question of whether generally there may be this much "late" user innovation in S-RET and other already relatively stable and mature technologies. In Chapter 3 we identified that when a high diversity of user needs and settings prevails, areas of (gradually expanding) need space become too small for producers to notice or to yield required profits and are thus remain unserved by producers. This then results in continued unmet user needs that give rise to modifications and inventions by users (see Chapter 3, Figure 3.3).

Regarding the generalizability of this pattern, we can discern two analytical interpretations. The first interpretation is that the protracted blending of innovation and diffusion could simply now be more visible thanks to the traces the innovations currently leave in internet forums that would not have been there just a decade or two earlier to draw academic attention (Fleck, 1993; 
Hyysalo and Usenyuk, 2015). The second, and complementary, interpretation is that these hybrid internet forums in themselves catalyze some user innovation relatively late in the process of sociotechnical change through providing peer knowledge exchanges, peer encouragement, and a peer learning environment that pushes some users toward a deeper engagement with technology and toward adaptations, modifications, and innovation. In this interpretation, the unmet user needs would remain unmet if users did not get peer encouragement and support to meet their needs with their own solutions. Whichever the case the findings underscore the need to pay close attention to the "third generation of research" on user contributions arising from increased digital connectivity (outlined in the introduction) also in the energy sector.

The studied internet communities also underline a hitherto undiscussed form of social value generated by user innovators, namely in-depth knowledge production and sharing among greater peer networks. Our analysis suggests that in the presence of large-scale internet communities this may in fact produce greater social value than that resulting from the diffusion of user-made innovations. As discussed in Chapter 3, the direct diffusion of S-RET user innovation happened through manufacturers, users turning into entrepreneurs, and through peer adoption, but only in somewhat modest numbers. More user innovations were diffused by spurring on "innovative diffusion" resulting in further projects and adaptations to technology, which blends adoption and further inventive modifications. These projects and their sharing among peers build a technological peer culture among citizens, turn many user innovators user-side intermediaries, and aid market creation and legitimacy creation for the alternative new technology.

\subsubsection{The key differences between wide-reaching and diverse Innovation communities and local communities}

There are important differences between early local community forms and those that accompany wider proliferation and continued innovation by users. Unlike location-independent and diverse-orientation internet communities, the communities of deeply committed enthusiasts are not necessarily able to, or even likely to, grow into a substantial force in the later stages of sociotechnical change. Firstly, the locally anchored enthusiast communities face challenges in changing participant orientations to serve other users rather than advancing their own projects. Across community domains, the deeply knowledgeable community members have been found to soon tire of giving advice to evernew novices. Communities that feature greater diversity in orientations and activities also have a wider set of participant positions, which is conductive to providing the requisite help and to build a critical mass in membership (Freeman, 2007, 2015; Johnson et al., 2010). Secondly, in technologies such as S-RET supporting mass adoption is tied to the capacity to address the variety of settings in which the technology is used, the purposes it is used for, and the ways in which it is deployed-which all require mechanisms to effectively 
gain, transfer, and pool a knowledge base related to different locales, practices and preferences. It is not trivial for local enthusiast communities to create these, and if they do not, the problems encountered by diverse new adopters, adapters, and inventors will not be alleviated as peer responses simply remain too slow and too superficial to guide the help-seeking users in moving forwardwe also saw this in Chapter 4 (see also Murto et al., 2019a).

Concretizing the above regarding energy communities, community energy and internet-based new forms of energy communities provide differing and differently prospering channels to aid the proliferation of S-RET. Localitybound community energy initiatives premised on shared finance and shared produce (Devine-Wright and Walker, 2008; Creamer et al., 2018) are likely to face a steep barrier when aiding the wide spread of S-RETs: the local energy communities, even local grassroots innovation communities, are unlikely to have the requisite knowledge base and resources to help hundreds of thousands of others in their adoption- and development-related questions and concerns. Their capacity to do so could potentially be improved through pairing local community energy initiatives with internet discussion channels. But again the question remains about whether the typically ideologically motivated grassroots community participants have the wide variety of in-depth technical knowhow, as well as the motivation, to help differently motivated mainstream users and whether they would be willing to invest in the basic running costs, moderation, and site maintenance all this takes (cf. Smith et al., 2016b). The present book thus acknowledges the value of community energy as an important type of adopter setting and as a means to foster energy democracy locally, but it remains critical regarding the capacity and willingness of community energy initiatives to sufficiently support the wide proliferation of S-RET.

Concretizing the findings regarding user-innovation communities and diffusion support they may provide results in somewhat similar reasoning. Von Hippel has argued the case well for why user innovators do not invest in diffusing their innovations to the degree that their social value would justify unless they turn to user-entrepreneurs (von Hippel et al., 2017; De Jong et al., 2015). While we agree with the above reasoning, the framing should be expanded to ask what community forms may then be conducive to the diffusion of the technology alternatives produced by users?

Hybrid communities feature a mix of innovating and non-innovating users and exchanges about innovation, as well as just practicing, and in doing so they feature a wider variety of orientations. From technical sports-mountain biking, rodeo kayaking, moth sailing — we know that effective user-innovation diffusion happens among peers (Luthe et al., 2005; Hienert, 2006; Hyysalo, 2009; Hienert et al., 2014; Gambardella et al., 2017; Bethwaite, 2008). Common to these domains is the frequency of events and competitions, which creates spaces that are not focused on innovation per se but on practicing a sport or hobby (Hyysalo, 2009; Bethwaite, 2008). In such spaces, solution adoption happens through the imitation of winners - some of whom have invented or use the inventions by other users. Adoption also happens through discussions 
and advice given among peers on the latest developments, which includes user innovations and modifications, and is not limited to the discussions held among the relatively few user innovators. The key point is this: the hybrid community forms bridge several local communities (where more innovationdirected activities take place) and offer a setting where the benefits and makeup of all novelties are discussed as it is pivotal to the competent practice of the sport, and the latter process remains agnostic to whether they emerged from a producer or from a user. It is this wider diffusion-facilitating propensity that renders these hybrid forms of community effective in diffusing user innovation as well. ${ }^{1}$

Competitive high-tech equipment-reliant sports are of course particular domains. The diffusion propensity in a domain grows lower if the imperative to keep up with technical advances is less acute or if the diversity of needs is even greater, so that emulation of solutions by others does not yield similar benefits (Hyysalo and Usenyuk, 2015). The S-RET internet communities studied in this book exemplify a setting where these damping effects are in place-but which nonetheless allows otherwise geographically separated people to learn from each other-and a setting which is conducive for the general diffusion of S-RETs. As discussed in Chapter 3, the forums do not provide good support for the direct copying of the innovations made by peers and, consequently, feature somewhat limited direct adoption in a comparison of adaptive copying in DIY projects, again for both user- and producer-originated solutions. The diffusion of user-generated solutions could be facilitated by improved means for copying, a higher uniformity of needs, or more acute benefits from improvements to users. Regardless of the shortcomings of copying, the forums may be more powerful as user-innovation diffusion agents than a pure user-innovation community might be, for instance, the DIY section of a forum viewed in isolation or a more innovation-prone forum, which our interviewees noted as just lacking critical mass even for effective innovation support. It is further noteworthy that the capacities of communities to facilitate diffusion can, and often have, become curbed through moving the internet communities from open discussion platforms to "walled garden" settings, such as WhatsApp or Facebook groups, which do not support active moderation and archiving as effectively. Again, all the above underscores the importance of digital mediation in user contributions to innovation and diffusion and, equally, the very different patterns it can take depending on the domain characteristics.

To sum up the findings regarding user innovation and user communities in sociotechnical change, their impact is not limited to just providing technological solution variety at the onset of sociotechnical change that is then exploited or discarded by the market as a selection environment. Instead, this book has evidenced continued user innovation in tailoring the technology to new settings. Furthermore, the influence of user innovators has been found to reside beyond the particular solutions in the competence building and peerto-peer interactions, which are amplified through in-depth inventive engagement with technology and spread widely through the reach created by internet 
communities. Regarding the community form, the capacities of hybrid communities to aid both peer and overall technology diffusion may be superior to those of pure-bred user-innovation communities, firm-hosted communities, or locality-based energy communities. The peer governance, diversity of competencies, and plurality of orientations present in internet communities boost their capacity to provide timely and useful peer advice and thus to act as agents that can aid adoption, adaptation, and more deeply inventive projects.

\subsection{The intersection of transition studies and S\&TS: tracing the series of configurational movements to understand how users shape sociotechnical change}

The observation that active citizen users widely contribute to sociotechnical change beyond innovation and modification leads us to transition research that provides a framework in which the range of their contributions can be discussed and linked to each other.

In our analysis of Finnish heat pumps, adoption and a growing market are the prime mechanisms that signal user activities to other niche actors, as well as to the regime and landscape actors, and to potential users still aligned with the existing regime. Adoption is, however, importantly facilitated by a range of more active engagements with the niche technology. Adaptation and championing become relatively common when subsets of users face the need to alter the equipment or their social and technical contexts. As noted above, DIY and user-innovation activities add to the solution variety and signal further design needs to resellers and manufacturers and provide deep-level competence for intermediation in user communities, allowing these to build up into major repositories of knowledge and encouragement that aid the installations, troubleshooting, maintenance, and so on that are related to the novel technology. Our analysis further underscores that user communities are conducive to playing a role in market creation as they create and make accessible the qualifying and contextualizing information that potential adopters need in their purchasing and installation decisions. Wide user communities further aid in policing other actors in the market. The accumulation of internet discussion posts and their tendency to relate to the technology in a naturalizing fashion further contributes to building legitimacy for a novel technology.

In Chapter 5 we showed how these different activities by users feed into each other and note that many of these activities - particularly intermediation, market creation, and the building of legitimacy — became intensified with the expansion of internet communities. Without such publicly available open media, the peer capacity to affect not-yet users who are still aligned with the earlier regime would have existed to a lesser extent.

The transition framing is thus valuable in helping to extend the time frame in which to study how users drive sociotechnical change, as well as to expand the scope of relevant activities to be attended to in understanding how sociotechnical change becomes shaped (cf. Smith, 2012; Schot et al., 2016; Meelen 
et al., 2019). At the same time, conducting a long-term investigation into users that is also deeply informed by S\&TS and innovation studies draws attention to the need to build more accurate and fine-grained conceptual registers and models for transition phenomena.

Transition models have, to date, mostly operated at the level of the "overall story" of how particular sociotechnical transitions happen and different country contexts have been regarded as variants to the pathways formed within the transition (Geels et al., 2016). The same orientation is visible in studies of users and citizens during transitions. Findings on historical transitions in specific countries are abstracted in order to build models at the level of overall transition (see, e.g., Kanger and Schot, 2018; Kanger et al., 2018) and more in-depth studies on moments and sites of current sustainability transitions are equally treated as contributing to the overall transition models, whilst these deeper studies are almost exclusively from global early mover settings (see, e.g., Meelen et al., 2019; Truffer, 2003; Ornetzeder and Rohracher, 2013). These studies are then taken implicate that once the niche technologies mature to a point where the acceleration phase begins, they will then proliferate to societies in ever-widening numbers (Geels and Schot, 2007; Schot et al., 2016; Kanger and Schot, 2016), factually repeating the assumptions made in the (early) diffusion of innovation research (Rogers, 2010), with the addition that transition research stresses that the social-embedding process-changes in institutions, cultural image, consumer behavior, market models - is needed for the technologies to proliferate (Hoogma et al., 2002; Kanger et al., 2018).

But the unified overall story built on studies of globally early settings may not well represent the localized transitions where "rubber meets the road" if transitions to sustainability are ever to happen worldwide. The early-follower and later-mover settings interface with the alternative solutions that are already in a more developed phase technically regarding commercialization, distribution, and usage patterns. At the same time, the later-mover settings are likely to feature characteristics that differ from the overall story, as well as early development contexts regarding geographical conditions and the institutional environment (Truffer, 2003; Meelen, 2019; DeWald and Truffer, 2012; Meelen, Frenken et al., 2019), market conditions and differences in technology characteristics (Ornetzeder and Rochracher, 2013; Nielsen, 2016), local technology competition (Lovio et al., 2011), and issues of wealth and inequality (Smith et al., 2016); and dynamics among the users. Our analysis of S-RETs in Finland in the 2000s underscores how the overall story-level depictions can also miss out the emerging phenomena that later adopter settings may enjoy. A good example is the amplification that digital connectivity has introduced to the citizen's capacity to network, build communities, and to affect sociotechnical change.

The BOAP line of study subsequently suggests reconceptualizing users in sociotechnical change through two concepts: innofusion and a series of configurational movements. Innofusion means the development of a range of sociotechnical characteristics during diffusion, including significant improvement to the 
technology characteristics (Fleck, 1993; Heiskanen et al., 2014; Hyysalo and Usenyuk, 2015). The concept stresses protracted re-innovation in the product and system characteristics in response to varying and newly surfacing local requirements and opportunities. At the heart of this concept is the observation that technologies do not spread in a vacuum but face different environmental, market, institutional, and cultural conditions in new country, locality, and organizational arenas. Some smoother adoption and limited re-innovation of the technology tend to run in parallel in sites that are already better aligned with the new technology (Rogers, 2010; Mignon and Bergek, 2016). ${ }^{2}$ In this view, the zoomed-out view of a whole system transition (as an expanding technological niche and its social embedding) is simply not how the myriad of localized transitions play out, and at the end of the day, it is these localized transitions that comprise the overall change. New ways to depict transition processes are hence needed.

The innofusion process, in turn, features series of configurational movements-key biographic moments associated with shifts in the arenas and associated ecologies of actors - in which the material, social, and cultural characteristics of technology become structured and restructured (Hyysalo, 2010; Hyysalo et al., 2019a; Glaser et al., 2020). These movements typically start in more informal, fluid and rapidly changing configurations and gradually lead to a normalized or "cold" sociotechnical order where changes in technology characteristics, identities of users, and types of use, as well as the institutional environment, remain incremental (Pfaffenberger, 1992; Callon, 1991, 1998).

Tracing the series of configurational movements provides a lens through which to attend to the specifics of how new technology becomes socially and materially shaped in the course of sociotechnical change. Doing so helps identify the shifts and sites that have been consequential in the sociotechnical change, offering concrete points to look for in other localized transition processes and potentially supporting them. Examining heat-pump proliferation in the detached house market to about $80 \%$ of maximal market penetration in Finland - a country that was at first an early adopter but, after its initial market collapse, fell into an early-follower position-allowed us to draw out nine key configurational movements in Chapter 5 and to abstract a minimal set of configurational movements that occur in a successful follower country transition:

i. The discovery of underlying technology principles and design of basic solutions take place elsewhere; activities from both suppliers and users potentially feature.

ii. Initial domestic introduction and adoption result in a nascent ecology of actors, featuring activities from both suppliers and users and, potentially, various third parties.

iii. Adaptation, championing, and user innovation take place so as to better suit the technology to the consumer specificities in the country. These activities are primarily conducted by users. 
iv. The emergence of intermediation that lays ground for effective domestic market, which potentially features activities from suppliers, users, and third parties and can be dominated by any one of the actor group.

v. Further technology and market development elsewhere feed into the development of the domestic market (through, e.g., lowering prices, improved logistics, improved reliability, etc.), while the domestic market may also affect some of the technology development elsewhere, resulting in better-tailored systems for the particular market. This movement features activities from suppliers and potentially from third parties and users.

vi. The emergence of mass-market conditions and maturation of the industrial field in the country; activities are driven by suppliers, while users and Intermediaries play roles as well.

vii. There is an interplay with adjoining technologies and spin-outs to new sociotechnical configurations; users, suppliers, and third parties are all likely to be active.

We further note in Chapter 5 that some of these configurational movements are sequential $(1,2,3,6)$ whereas others may appear in parallel $(4,5,7)$, depending on biographic sub-trajectories, which may become reversed in the course of how the sociotechnical configurations and ecologies of actors develop. In Finnish heat pumps, for instance, we see a contingent "early curbing and death," "second introduction," "acceleration," "supporting intertwinements and maturation," and "spin-out biography" phasing.

Analyzing transition as a series of configurational movements calls into question some of the overall story assumptions, particularly those regarding the acceleration phase of transition. The abstracted set of configurational movements above and our later analyses of energy retrofits in housing companies (Murto et al., 2019a, 2019b) suggest that the smooth acceleration associated with the speeding up of diffusion and well-functioning markets for the new alternatives emerge quite late in the transition process. While the basic niche characteristics are built up during the take-off, the alternative novelties still proliferate against the grain of the market and institutional conditions set by earlier technologies for a considerable time. The acceleration phase might thus be split roughly in half, into a phase of "widening proliferation" and one of "diffusion through markets," which feature importantly different characteristics, policy responses, and managerial implications (see below for the policy and managerial implications).

Overall, this book argues that the study of users and citizens in transitions - similar to many other intricate transition processes that tend toward invisibility, such as learning ${ }^{3}$ - is not adequately addressed if only patched together from piecemeal case studies or from broad overviews that rest on limited empirical coverage. A recalibration of transition research is in order to address both the detailed processes and their long-term interconnections without losing the relevant details in a too zoomed-out view for the sake 
of gaining clear and easily graspable models (Farla et al., 2012; Zolfagharian et al., 2019; Murto et al., 2020). The BOAP framework is one candidate for the task of bridging the needed detailed ethnographic studies to the historical evolution.

\subsection{A user-domain focalized BOAP investigation: reflections}

The focus of the BOAP analysis in this book has been on user activities in the course of a long-haul sociotechnical transformation. The S-RET systems studied present an "industrial field of medium complexity," It is, for instance, considerably less complex and features far fewer arenas and smaller ecologies of actors than the organizational software studied earlier (e.g., Pollock and Williams, 2008, 2016). At the same time, it is considerably more complex than most consumer good fields (e.g., Baldwin et al., 2006) as S-RET systems involve some permitting and other institutional regulation, require separate installers, are relatively costly, and have to integrate into very diverse housing settings and everyday practices.

The study has been purposively cast to focus on one country and one group of technologies, with an added weight on heat pumps that, thus far, are the most widely spread of the S-RETs in Finland. The data gathering has been a mix of ethnographic and historical analysis typical of BOAP, and similarly a mix of several more detailed foci, wider overview analyses, and quantitative and qualitative analysis. The focus on sociotechnical change in one country context and user activities has meant that wider "global" changes were examined as an endogenized context, that is, it was included insofar as these developments have had a bearing on the national and local developments. The same goes for various neighboring developments to the S-RETs in Finland during the extended study time of about 30 years - they have been covered from other research and secondary literature and then linked to the focal analyses insofar as they are relevant. The approach presents a new way to contextualize user activities as well as to focalize long-term sociotechnical change research onto mid-range phenomena.

The present book features a one-side biased biography of a sociotechnical change in that the arenas and ecologies of actors beyond Finnish user activities have been studied more thinly. In particular, the international context where most S-RET producers have become located in the Far East has not been studied first-hand. These limitations do not render the approach meek. BOAP has guided the researchers into attempting to trace the endogenized influences back to a national context and users, and guarded the analysts and readers from reliance on single snapshots or hollow-arch overviews, as well as from pretending that the present account tells the whole story. BOAP also points out the possibilities for further research by extending the study to the now implicated international arenas, as well as in continuing the Finnish context research through tracing the future trajectories of the renewable energy technologies, particularly because their installation types and scales are presently expanding 
from residential systems to a larger community and industrial-scale deployments, which at once shifts both the relevant arenas, the ecology of actors populating them, and the sociotechnical configurations involved.

The present book further elaborates on the dual orientation that characterizes most BOAP research: on the one hand, as (just) a methodological guideline and, on the other hand, a methodology that becomes (re)connected to a more encompassing theory tradition.

In the present book the status of BOAP was first that of a methodological approach to building and extending the research design. In this capacity and long into the accumulation of the empirical work, the findings would have been compatible with several different theory frames. BOAP as a methodological approach tied the analysis to the study of emergence, the more durable structures in place, and their transformations, and thus forced the analysis beyond a bird's-eye overview of systems change, a focus on particular topics (such as user innovations), or making a snapshot study of actor networks in one locale related to S-RETs. The additional merit of using BOAP as "just" a methodological guide is its capacity to steer towards theory bridging and interrogation, which was suggested as the ninth typical feature of BOAP studies in Chapter 2. The stringing of the sub-studies typical to BOAP leads analysts to relate to the substantive research traditions and their research designs that are relevant to the sub-study phenomena. In the present book the relationship to these theories has been appreciative, yet their juxtaposition and empirical insights have qualified each theory tradition with respect to what they cover and what they leave unattended (see the above summary findings). In this capacity, the BOAP methodology has a propensity to create a double epistemic inquiry: one related to the research domains and another related to the theories, study framings, and research designs that have been used to study the research domain.

A second orientation to BOAP emerged in the later years of the decadelong study on user activities in S-RETs in Finland. The status of BOAP moved into one that was (re)connected to substantive theory tradition in ecological sociology once it became important to better understand the ecology of actors, and user contributions to intermediation and market creation. Similarly to earlier work on health care technologies (Hyysalo, 2010), where the study was explicitly informed by activity theory and symbolic interactionist S\&TS, also here the symbolic interactionist social worlds/arenas framework guided how the activities of peer communities and ecologies of intermediaries were linked to ecologies of actors, the emergence and stabilization of arenas, and endogenized causation to arenas elsewhere. This (re)connection to substantive theory helped both the theoretical sampling in the final years of the BOAP investigation and in giving a final interpretation of the results.

It is important to notice these two orientations to BOAP could also run on colliding courses. If the connection to any one theory tradition was strong from the outset, an appreciative theory bridging BOAP sub-study framings 
would be unlikely to happen, and instead a single competing theoretical account would emerge. Conversely, if the BOAP was only used strictly as a methodological guide and theory only informed the final interpretation of the gathered results, it is likely that the methodology and methods will not have sufficiently informed the data gathering so that the issues important to that theory would be gathered. In the present book middle ground was sought between the merely methodological and theory-connected use of BOAP.

Regarding generalizing from a BOAP investigation, a careful reader might have noticed that there are two implied avenues for generalization in the present study. First, the sub-studies on user innovation, on user activities in the energy transition, and on the nature of technology-oriented peer communities link to wider bodies of research on the same topics, and results from Finland (which is in many respects a typical Western industrialized country that has identifiable contextual and energy economics features that help comparing findings across countries; see the Appendix) and its S-RET-related phenomena add to the stock of findings that offer generalizability in these bodies of research.

Second, beyond the sub-studies, the investigation pursued through the BOAP methodology offers a generalization on sociotechnical change dynamics that is premised on deep and extended lines of enquiry, which traverses between highly situational snapshot studies and a broad overview and system change accounts, which tend to rest on hollow-arch empirics. Metaphorically speaking, it provides construction beams that aid linking more short-term or more superficial studies to one another or, to use the metaphor of quilting, it provides long pieces of canvas and long fibers that give strength and structure so that shorter pieces can be added in. The net importance of the latter generalization strategy is that BOAP-type investigations can provide needed empirical grounding to "middle-ground theory" building of sociotechnical change phenomena, particularly on topics that tend to remain invisible in official statistics and other record keeping that require empirical primary studies.

\subsection{Facilitating user-driven energy transitions: managerial and policy implications}

\subsubsection{User-driven energy and energy technology business}

Energy systems have traditionally presented one of the most centralized and supply-side oriented environments to the extent that consumers are commonly referred to as "the demand side" and supplier offerings of power and heat feature remarkably small variations. Climate change and the ensuing imperative to transition to fossil-fuel-free-energy systems are rapidly changing all this. It is widely recognized that energy companies need to change their business logics from being the carbon problem to resolving the carbon problem. Doing so implicates that companies in the energy sector must also become customercentered and engage in user-driven transformation as consumers require new 
services and options. In turn, climate and energy policy should ensure that this is properly incentivized and sanctioned. But what exactly does this user-driven transformation entail?

The analysis in this book suggests that most of today's customer-centered actions by energy companies are still best characterized as seeking to develop solutions for potential customers, including:

- increasing the diversity of services from just two to three pricing options to options for different energy sources and mixes;

- introducing energy plans with two-way sales for prosumers;

- introducing options to be part of demand-response measures through virtual power plants;

- introducing options for renting or leasing solar panels or other renewable production;

- providing more sophisticated energy monitoring devices and interfaces;

- providing more sophisticated building automation (and monitoring) services to reduce energy consumption;

- featuring evaluation services to assess where energy efficiency could be improved in the house;

- energy-use reduction as a service (ESCO), where energy efficiency measures are billed from the differential of reduced heating bills over time;

- building area-wide solutions for heat/cooling and power storage to handle intermittent production and fluctuating prices, and to provide better services for customers.

All these are highly necessary for energy transition. But from a user-driven innovation and business perspective, these are not far going or very transformative renewals. In fact, such measures represent the energy sector as barely on a par with business-as-usual in most other industrial sectors. Being "user-driven" boils down to added market segmentation and service differentiation to offerings, and using service design to ensure the new services and products feature sufficient user experience (Hyysalo et al., 2016a; 2016c).

Our analysis of S-RET systems in Finland shows that once consumers gain the possibility to choose and operate their own energy systems, they grow more active, just as they have done in other domains such as ICT. This implies that energy technology providers and energy companies can move towards a deeper user-driven orientation in their strategies and operations, one that entails not only designing for customers but also designing with customers and engaging with the development done $b y$ customers (Prahalad and Ramasvamy, 2004; von Hippel, 2005; Hyysalo et al., 2016). Such a portfolio would minimally entail business offerings such as:

- providing a platform for and offering a pooling service for establishing energy communities between neighboring residential buildings, which would provide active energy citizens with more flexibility in balancing 
their prosumption profiles and would help achieve larger community energy systems; the energy company would gain commission and potentially the energy contract from the community;

- venturing into energy retrofit business to build deep retrofits and financing them as a "one-stop shop" with the energy service company business model;

- $\quad$ acting as a broker to pool willing houses or housing companies to form an energy community and to achieve network effects in deep retrofitting, effectively supporting the retrofit venturing;

- hosting or supporting internet communities in emerging areas, such as deep retrofits, hybrid heating systems, and energy communities; the benefits would accrue from learning about the market and helping it grow, similarly to what has happened to the residential S-RETS studied in this book.

As careful readers recognize, the above suggested 'with' measures necessarily feature business partnerships and the diversification of business for energy and energy technology companies, particularly into energy retrofit markets. An underlying condition is the acceptance of the ambidexter business model, where traditional energy companies accept volume loss in their traditional heat and power sales to existing customers and compensate for it with new business that brings rents from building higher energy efficiency and from the attraction of new customers. In this line of thinking, the energy company or energy technology company is foremost out to serve its customers' active or potential initiatives in achieving a lower and more renewable energy production profile. The financial benefits follow from being a pioneer in pursuing deep customer orientation and gaining brand recognition for advancing the green transition. There is thus a considerable difference to a "for" orientation, where these companies still intend to push for more sales of their products even if through more diverse and additional services-inclusive business model.

The rationale for underscoring the deep user-driven transformation is that S-RET installations, deep retrofits, hybrid systems, and pooled energy communities are still in their globally nascent stages and the difficulties in acquiring them curb most customers from taking action (Murto et al., 2019a, 2019b). And, similarly to residential S-RET in the Finnish context two decades before, there is a strong latent demand premised on environmental benefits and positive cost-effectiveness. Such conditions arguably lead to these markets becoming formed at some point-either through entrants, via more piecemeal solutions, and/or through the strategic actions taken by some forerunner incumbent companies.

Moving yet deeper into actions taken by consumers in transitions, our results underscore three managerial implications. First, energy and energy technology companies could shift to open user innovation orientation in gauging and seeking to help those citizens and citizen groups that try to create new sustainable energy solutions. Not all business and innovation wisdom recides within 
the companies and being involved in experiments lead by citizens offers first mover opportunities to learn and develop further both technical and business solutions. Second and related, monitoring user modifications and innovations may reveal points for innovation for technology manufacturers. User alterations reveal both need and solution information and can be analyzed regarding both (von Hippel and von Grogh, 2016). The information on needs was previously difficult to highlight, but with internet communities, user problems, wishes, alterations, and innovations it is now readily available for producers to follow (Grapher and Ibert, 2013; Johnson et al., 2010; Hyysalo et al., 2015). Regarding solution information, many user innovators are happy to freely reveal their solutions, and companies could also volunteer to further develop the best DIY ideas with a fair compensation scheme in place-the sums users ask for are commonly low if they even ask for a sum (von Hippel, 2005, 2016). Our results indicate that it may make the best sense to monitor where the solutions are clustered and what kind of adoptions and innovation pathways they form together as this articulates demand and points to improved commercial systems, thus helping to move beyond the problems of isolated solutions to very particular user needs (see Figure 3.3; Nielsen et al., 2016). Thus, on entry to emerging energy technology markets, producers would benefit from following the interaction arenas where users discuss the technology and display their problems and solutions.

Third, companies or industry associations may wish to sponsor user-run internet communities that feature effective archiving and moderation. Even as some of their actions, such as policing the field against poor installations, may be irritating in the short haul, there is a clear market organizing and legitimacy-creating effect over the long haul. These effects will not, however, come to fruition only through Facebook groups or other non-moderated and ineffectively archived social media. Without moderation, an internet community can quickly grow useless for all concerned through, for instance, false claims, tarnishing campaigns, and libel content. Without archiving, prospective buyers and users will not be able to effectively find the information they need.

\subsubsection{User-driven energy and climate policy}

For policy action, the core finding of the analyses in this book is that it takes a long time before effective market and informational mechanisms accompany supplier and user solutions in such a way that there is a well-functioning market for new energy technology. The cumulative sales figures can run into tens of thousands of units, yet the ecology of actors may still remain ineffective in pushing low-carbon solutions for true mass adoption. The common policy reaction is to focus action on the supply side, and to provide relatively minor and undifferentiated subsidies to the demand side. This is commonly paired with only gradually changing regulation and other institutions in order 
to better support the new alternatives, typically through processes slowed by lobbying by incumbent businesses.

Our analyses lend support to the view that new alternative more sustainable solutions face obstacles and hindrances due to having to operate in a market and institutional environment built for the technologies they would replace (e.g., Hoogma et al., 2002; Murto et al., 2018a; de Rubens et al., 2018). Various hindrances in, for example, permissions, regulations pertaining to pricing, thresholds set for subsidies, taxation, etc., may continue well into the proliferation process (Heiskanen et al., 2014; Berninger et al., 2017). When these are paired with the information and productization deficiencies that are common to the early phases of a new technology type and with missing market actors and market information, the summative damping effect imposed on adopters can be considerable. Environmental policy should thus be more deeply intertwined with innovation and technology policies (see below) and take the need to monitor the formation of effective markets for more sustainable alternatives seriously - and be ready to support it. The support measures can happen through removing unnecessary or poorly motivated market hindrances as well as through setting up experiments in which more level playing field is provided as well as through public or public-private intermediary actors to support the emerging niche markets once they have shown their potential yield to environmental benefits.

In addition to supply-side and market-structuring measures, the present analyses underscore that users are more active across the long process of sociotechnical change than the mainstream policy advice notices, and their capacity to contribute to advancing sustainable sociotechnical change can be supported beyond incentivizing adoption.

First, recognizing citizens as active and well-informed actors calls for a rescaling of climate and energy policy from the present top-down one to one that actively responds to and facilitates initiatives on the ground (Jalas et al., 2017). Finland alone features several citizen-initiated climate actions along with internet forums that directly evidence need and enthusiasm. These feature a carbon-neutral municipality network in which presently over $30 \%$ of the country's population has joined and, at a much finer level of engagement, housing company clubs that pool housing companies for energy retrofits. In both cases, climate policy actors have joined and come to facilitate and provide longevity to the initiatives. The main locus of action in responding to such measures is likely to reside in municipalities and areas, but also national bodies In Finland energy efficiency agency Motiva, have shown themselves capable of recognizing and responding to such initiatives. Providing funding for running and expanding such citizen initiatives and linking them with technology experiments and legislative trials are strategies that are already pursued in many countries and clearly should be pursued even more in the future.

Second, under the conditions of sector-wide transformation, energy, innovation and environmental policies should be pursued in tandem (Hyysalo et al., 2019b). The policies should be further linked to cross-sectoral measures 
to remove the typical mesh of existing regulation that unnecessarily blocks or hinders citizen choice and engagement with new types of technologies ranging from specific items related to permitting, taxation, ownership rules, actor responsibilities, and so on that have been formed under the conditions of the previous technology base. This calls for the active monitoring of changing sociotechnical conditions and typically calls for active engagement with both citizen communities, and intermediaries and researchers who work with the cutting-edge change. Such action can be rendered anticipatory through multi-stakeholder arena processes (Hyysalo et al., 2019a, 2019b; Lukkarinen et al., 2020).

Third, peer support among consumers is a valuable resource that could be cultivated. Peer support can foster deepening engagement with energy technology that leads to higher consciousness of how much energy is consumed and how it has been produced. With regard to facilitating internet communities themselves, it may be illusory to seek to create such discussion forums through policy measures, but allowing (often anonymous) discussions to prevail holds benefits, and some energy and climate policy actors, such as energy councilors, might offer their advice through these discussion forums, as has already happened in youth work after the realization that nowadays it is increasingly on the internet where people can be reached (Hyysalo and Juntunen, 2018). Some forums may benefit from nominal support in order to cover running costs (see the above regarding corporate sponsorship).

Importantly, peer-to-peer advice, local energy communities and internet communities should be regarded as important and thus be explicitly assessed when passing regulation. For instance, measures to counter libel content on the internet by removing all anonymity could also wipe out positive anonymity in internet discussions, whereas displayed anonymity that is traceable to real verified persons in cases of libel action would not. Also, the nature of commercial internet plans available to consumers should be monitored: open internet should be retained as a possibility in open society to avoid lapsing into the availability of only "walled garden" ecosystems by leading commercial providers as this can severely limit the effective forms of digital citizenship available. These arguments are part of innovation wetlands-thinking (Torrance and Von Hippel, 2016) in that diversity and exploration by citizens is a valuable but unflagged resource that is easily erased, similarly to how seemingly useless wetlands have come to be recognized as hotbeds for biodiversity and their erasure requires consideration. Needless to say, the same goes for consumer innovations and DIY activities.

Regarding user innovations, policymakers commonly view them as too isolated and particular for targeting policy action (Nielsen et al., 2016). As indicated above with managerial measures policy action could target areas where clusters of user innovations are occurring and build programs to foster producer-user interactions in order to develop the user improvements into commercialized offerings. 
This said, we should reflect on the limits to policy action such as the limits to what policy measures can do to counter the potential under-diffusion of consumer innovations in S-RETs. As argued in Chapter 3 innovating consumers would have the incentive to take the time and energy to document their often complex hacks and designs for others to easily adopt them. The innovating consumers could, however, be motivated to do so if there were effective platforms and templates available for the effective display of their problems in such a way that it would help to present them and, as a side effect, make a more structured information package out of the display (e.g., if an easy way to make blueprints was available or if there was an easy way for the user to point to the exact location of the modification and make parts lists). But what could policy provide? Not much apart from funding a research program or potential challenge competition aimed to create better sharing platforms for physical citizen designs.

Throughout the course of this book we have shown how activities by citizens can turn many core assumptions about energy system change upside down. Ordinary people innovate, they structure markets and, gradually, their purchases can drive a wholesale change regarding heating practices and, by implication, can also power their own lives with an S-RET that generates electricity. These processes may benefit from policy intervention, but foremost they may only require the absence of policy blocking. Similarly, technology development and mass-manufacturing economies are required to get the technology options to the point of rough cost-effectiveness, but again these must not all be perfect before the innovation-in-use kicks in and brings in further improvements. Incumbent energy companies and energy technology providers may either join or seek to initially curb the process but would be fools to think they would have the power to stop citizens from wanting and driving the transformation of energy systems in the long haul and across country contexts.

\section{Notes}

1 Needless to say, the exact dynamics would merit careful studies of the diffusion and recommendation behaviors in such communities.

2 Innofusion is likely to be more common with S-RETs that are deployed in wide and variable ways, at different scales, and with different ownership models (such as is the case with heat pumps, energy efficiency renovations, or pellet and solid-wood burning) than in S-RETs that require permitted and uniform installations, such as community-scale wind power (Juntunen and Hyysalo, 2015).

3 See Chapter 1 and Chapter 5: These processes are invisible because they are not recognized by the actors experiencing them as occurring or being of importance; they are consequentially not systematically recorded and do not reliably show in easily accessible data series or in using standard research instruments. Any one of these conditions being present leads to a requirement for considerably more arduous research designs than is customary in mainstream transitions research. 